\title{
Comparative study on the broadening of exciton luminescence linewidth due to phonon in zinc-blende and wurtzite GaN epilayers
}

\author{
S. J. Xu, ${ }^{\text {a) }}$ L. X. Zheng, S. H. Cheung, M. H. Xie, and S. Y. Tong ${ }^{\text {b) }}$ \\ Department of Physics and HKU-CAS Joint Laboratory on New Materials, The University of Hong Kong, \\ Pokfulam Road, Hong Kong, China \\ Hui Yang \\ State Key Laboratory on Integrated Optoelectronics, Institute of Semiconductors, CAS, \\ Beijing 100083, China
}

(Received 10 June 2002; accepted 9 October 2002)

\begin{abstract}
Linewidth broadening of exciton luminescence in wurtzite and zinc-blende GaN epilayers was investigated as a function of temperature with photoluminescence. A widely accepted theoretical model was used to fit the experimental data, so that the coupling parameters between exciton and acoustic and longitudinal optical phonons were obtained for both structures. It was found that the coupling constants of both exciton-acoustic optical phonon coupling and exciton-longitudinal optical phonon coupling for zinc-blende $\mathrm{GaN}$ are almost twice as much as the corresponding values of wurtzite GaN. These results show that the relatively strong exciton-phonon scattering seems to be characteristic to zinc-blende GaN film. (c) 2002 American Institute of Physics.
\end{abstract}

[DOI: $10.1063 / 1.1526450]$

It is known that GaN exists in two different crystalline structures under usual conditions. The equilibrium phase is wurtzite $\mathrm{GaN}(\alpha-\mathrm{GaN})$, while the metastable zinc-blende $\mathrm{GaN}(\beta-\mathrm{GaN})$ can be expitaxially grown. In the past a few years, the structural, optical, and electrical properties of $\alpha-\mathrm{GaN}$ as well as $\alpha$-GaN-based devices have been extensively studied. ${ }^{1-3}$ However, many outstanding issues remain unsolved. Compared with $\alpha-\mathrm{GaN}$, fewer studies ${ }^{4}$ have been devoted to $\beta-\mathrm{GaN}$. This situation will change, as rapidly increasing attention has been paid to the system in recent years. ${ }^{5-9}$ Yang et al. ${ }^{10}$ and As et al. ${ }^{11}$ recently demonstrated electroluminescence from $\beta$-GaN $p-n$ junctions, which represents the starting point of $\beta$-GaN based optoelectronic devices. Theoretical work has predicted that $\beta-\mathrm{GaN}$ has different properties from $\alpha$-GaN, for example, smaller band-gap ${ }^{12,13}$ and easier $p$-type doping. ${ }^{14}$ Some of these have already been verified experimentally.

In this letter, a comparative study on exciton scattering by phonons in $\beta$-GaN and $\alpha$-GaN films is reported. The temperature dependence of the linewidth of exciton lines in the two GaN films is fitted with a theoretical model. It was found that the coupling strength of the excitons with both the acoustic phonons and the LO phonons in $\alpha$-GaN is much smaller than the corresponding ones in $\beta$-GaN. The relatively strong exciton-phonon coupling may thus be characteristic of $\beta-\mathrm{GaN}$.

The $\beta$-GaN films were grown on Si-doped GaAs (001) substrates by metalorganic vapor phase deposition using ammonia and trimethylgallium as $\mathrm{N}$ and Ga precursors, respectively. $\mathrm{H}_{2}$ was used as the carrier gas during deposition. A nucleation GaN layer was first deposited at $550^{\circ} \mathrm{C}$ for 10 min. It was followed by a $2-\mu$ m-thick $\beta$-GaN layer at $820^{\circ} \mathrm{C}$. The $\alpha$-GaN epilayers were directly grown on $6 \mathrm{H}-$

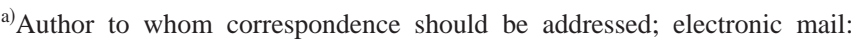
sjxu@hkucc.hku.hk

${ }^{b)}$ Present address: Department of Physics and Materials Science, City University of Hong Kong, Kowloon, Hong Kong.
}

$\mathrm{SiC}$ (0001) substrates at $650^{\circ} \mathrm{C}$ by plasma-assisted molecular beam epitaxy (MBE). ${ }^{15}$ The thickness of the $\alpha$-GaN epilayer was about $1.8 \mu \mathrm{m}$. A micro-Raman system was employed to characterize the phonon structures of the two $\mathrm{GaN}$ films at room temperature. The excitation laser source in the Raman scattering was the $514.5 \mathrm{~nm}$ line of an $\mathrm{Ar}^{+}$ laser. The incident laser beam is along the growth direction of the films and the back-scattering geometry was taken. The photoluminescence (PL) setup has been described previously (see Ref. 22). In the PL measurements, the same measurement conditions were applied to both samples.

Figure 1 shows the measured PL spectra from the $\beta$-GaN and the $\alpha$-GaN samples at different temperatures. At low temperature, the peaks at 3.269 and $3.151 \mathrm{eV}$ from $\beta$-GaN have been identified by many authors ${ }^{4-6,9}$ as the shallow donor-bound exciton and the donor-acceptor pair transition lines. Our varying excitation power experiment also presents a clear spectral evidence that the peak at about $3.151 \mathrm{eV}$ is the donor-acceptor pair transition. The linewidth of the donor-bound exciton line of $\beta$-GaN at $3.5 \mathrm{~K}$ is $14.6 \mathrm{meV}$, which is comparable to the best values reported so far. ${ }^{5}$ The linewidth of the dominant peak broadens to $54.9 \mathrm{meV}$ at 300 $\mathrm{K}$. To the best of our knowledge, this is the narrowest PL linewidth observed at room temperature for $\beta$-GaN. ${ }^{4,5}$ Moreover, the successful demonstration of electroluminescence from $\beta$-GaN $p-n$ junction ${ }^{10}$ also verified the high quality of the sample. In general, the shallow donor-bound exciton transition dominates the low temperature PL spectrum of $\alpha$-GaN. From Fig. 1, it can be seen that the linewidth of the dominant bound exciton line is $12.8 \mathrm{meV}$ at $3.5 \mathrm{~K}$, which is close to that of the $\beta$-GaN. The linewidth of the exciton line increases from 12.8 to $32.2 \mathrm{meV}$ when the temperature increases from 3.5 to $300 \mathrm{~K}$.

The temperature dependence of the linewidth for the lowest $1 \mathrm{~S}$ exciton transition in semiconductors can be given by ${ }^{16}$

$$
\Gamma(T)=\Gamma_{0}+\sigma T+\gamma N_{L O}(T) .
$$

The first term on the right-hand side is a constant, standing 

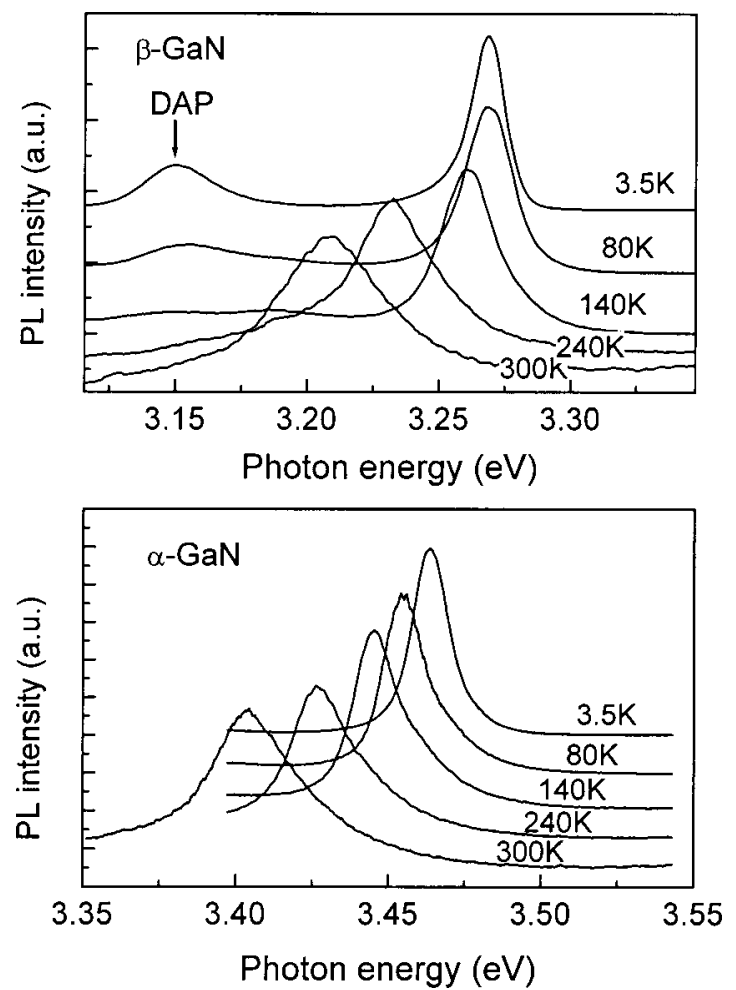

FIG. 1. Measured PL spectra of the zinc-blende GaN and the wurtzite GaN heteroepilayers at different temperatures. For clarity, all the spectra were normalized and were shifted vertically except one at $300 \mathrm{~K}$.

for the impurity and inperfection scattering. The second term arises from the scattering of the excitons by acoustic phonons, exhibiting the linear temperature dependence. $\sigma$ is the exciton-acoustic phonon coupling strength. The last term is due to the interaction of the excitons with the LO phonons and is proportional to the Bose function $N_{\mathrm{LO}}(T)$ $=\left[\exp \left(\hbar \omega_{\mathrm{LO}} / k_{B} T\right)-1\right]^{-1}$ for LO-phonon occupation, where $\hbar \omega_{L O}$ is the LO phonon energy and $\gamma$ represents the exciton-LO phonon coupling strength. The above theoretical model has been frequently employed to study the thermal broadening of exciton luminescence in various semiconductors. For $\alpha$-GaN, several groups ${ }^{17-19}$ fitted the experimental linewidth using Eq. (1).

Figure 2 shows the linewidth of the exciton luminescence in $\alpha-\mathrm{GaN}$ and $\beta$-GaN as a function of temperature. The solid lines represent the least-squares fitting of the data considering temperature-independent inhomogeneous broadening, acoustic phonon scattering, and LO phonon scattering. As a highly polar semiconductor, $\mathrm{GaN}$ possesses much stronger exciton-LO phonon scattering than other III-V semiconductors, such as GaAs. In order to accurately determine the characteristic energy of LO phonons in the $\beta$-GaN and the $\alpha$-GaN films, Raman scattering spectra of both samples were taken at room temperature, as shown in Fig. 3. The measured phonon energies of the $\alpha$-GaN and the $\beta$-GaN samples are in good agreement with the values reported in the literature. ${ }^{7,8,20,21}$

From Fig. 3, it can be seen that a low content of $\alpha$-GaN exists in the $\beta$-GaN host because of the resolved $\mathrm{E}_{2}$ phonon mode, which is a characteristic mode of $\alpha$-GaN. It should be pointed out that the $\mathrm{E}_{2}$ phonon mode is not always observable when we change the excitation spot. This implies that the low content of the $\alpha$-GaN phase in the $\beta$-GaN film is not

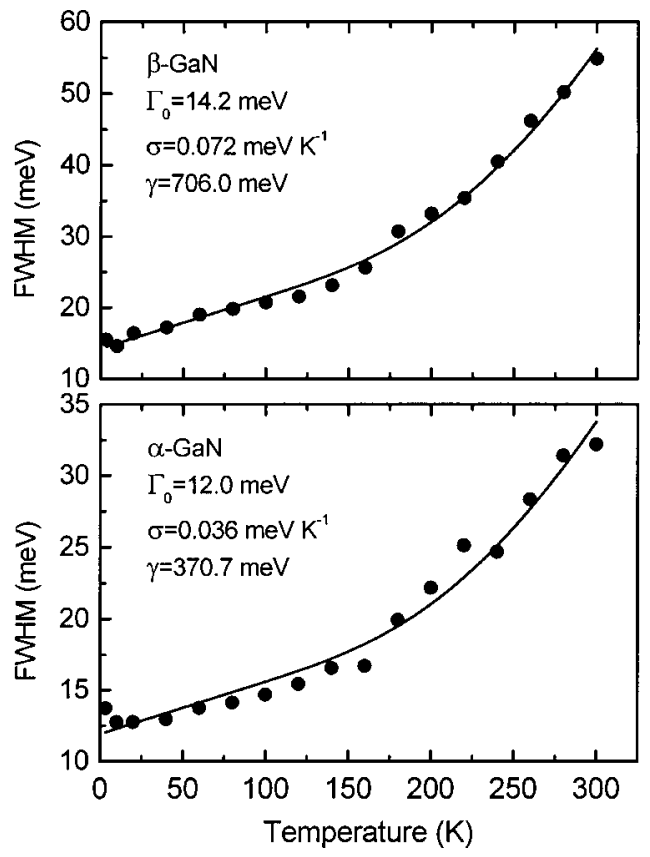

FIG. 2. Temperature dependence of the linewidth for two GaN epilayers. Solid lines are the fitting results with Eq. (1).

uniformly distributed. We took the measured energies of the LO phonons when fitting the linewidth in Fig. 2. It is found that the result is excellent with the following parameters: $\Gamma_{0}=14.2 \mathrm{meV}, \sigma=0.072 \mathrm{meV} \mathrm{K}^{-1}$, and $\gamma=706.0 \mathrm{meV}$ for $\beta$-GaN, and $\Gamma_{0}=12.0 \mathrm{meV}, \sigma=0.036 \mathrm{meV} \mathrm{K}^{-1}$, and $\gamma$ $=307.7 \mathrm{meV}$ for $\alpha$-GaN. It is noted that the $\sigma$ and $\gamma$ values obtained here for $\alpha$-GaN are consistent with those obtained by Buyanova et al. ${ }^{17}$ Obviously, the $\sigma$ and $\gamma$ values for $\beta$-GaN are much greater than the corresponding ones of $\alpha$-GaN, although their $\Gamma_{0}$ values are comparable. This suggests that there exists a stronger exciton-phonon scattering in $\beta$-GaN. Brandt and his co-workers ${ }^{4}$ also observed a large thermal broadening of the near-band edge transition in $\beta$-GaN. Furthermore, they pointed out that this was a characteristic property of $\beta$-GaN.

According to Rudin et al.'s theoretical calculations, ${ }^{16}$ the contribution of exciton-LO phonon scattering to the linewidth is mainly from the Fröhlich interaction. In general, the larger LO phonon energy favors stronger Fröhlich interaction

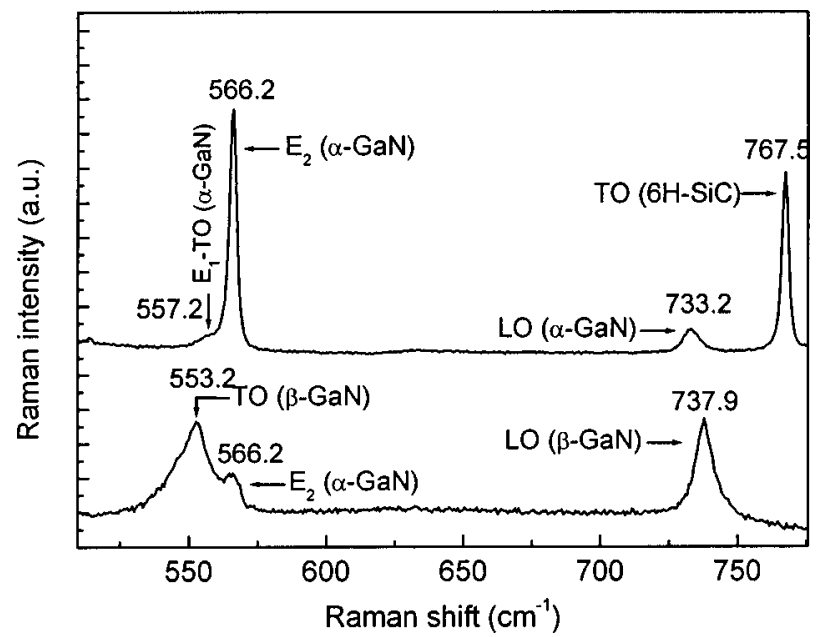

FIG. 3. Raman scattering spectra for two GaN epilayers at room temperature. 
and thus a larger coupling strength $\gamma$. From Fig. 3 we know that there is a slightly larger LO phonon energy for $\beta$-GaN than for $\alpha-\mathrm{GaN}$. This small energy difference is perhaps particularly important for polar semiconductors like $\mathrm{GaN}$ with very intense exciton-LO phonon Fröhlich scattering. Another important factor relating to the thermal broadening of the exciton lines is the binding energy of excitons. ${ }^{16}$ It is very likely for $\beta$ - $\mathrm{GaN}^{22,23}$ to have smaller binding energy of free excitons than $\alpha-G a N .{ }^{24,25}$ At higher temperatures, the free exciton recombination generally dominates in the PL spectra of GaN. This can be judged from the characteristic asymmetric lineshape of the free exciton emission. ${ }^{26}$ Note that the lineshape of the dominant emission line from the $\beta$-GaN film changes from asymmetric to symmetric when temperature is above $240 \mathrm{~K}$. But for the $\alpha-\mathrm{GaN}$ sample, the emission line keeps its characteristic asymmetric linshape from 80 up to $300 \mathrm{~K}$. This fact also indicates that $\beta-\mathrm{GaN}$ has relatively smaller binding energy for free excitons. It can be expected that $\beta$-GaN will have relatively broad emission line at higher temperatures. Therefore, both factors, the relatively larger LO phonon energy and relatively smaller free-exciton binding energy, will cause significant thermal broadening of the emission line in $\beta$-GaN, particularly, at higher temperatures.

At low and medium temperatures, the contribution of acoustic phonon scattering to the broadening of exciton line dominates. The exciton-acoustic phonon interaction takes place via the deformation potential and the piezoelectric coupling. The contribution of deformation potential scattering to the linwidth is approximately equal for wurtzite and zincblende $\mathrm{GaN}$ films due to the nearly equal deformation potentials of GaN with the two crystalline structures. ${ }^{27}$ However, the contribution of piezoelectric interaction is different. ${ }^{16}$ Thus, we believe that the difference between the excitonacoustic phonon coupling constants of $\beta$-GaN and $\alpha-\mathrm{GaN}$ is primarily due to different piezoelectric interactions for the two structures.

In order to further confirm the relatively stronger coupling of exciton with LO and acoustic phonons in $\beta$-GaN, we plotted the temperature dependence of the peak positions of the dominant emission lines for two GaN in Fig. 4. It is seen that the peak positions have almost same temperature dependence between $\beta$-GaN and $\alpha-\mathrm{GaN}$, but there is a shift of 195 $\mathrm{meV}$ in energy between the two. Note that the $\alpha$-GaN and $\beta$-GaN films investigated here were grown on different substrates. The same temperature dependence of the emission photon energies between the two GaN films provides an additional evidence that the relatively larger exciton-phonon scattering is an intrinsic property of the $\beta$-GaN epilayers.

In conclusion, the temperature dependence of the dominant emission lines from the GaN epilayers in wurtzite and zinc-blende structures has been compared. A widely accepted theoretical model was employed to fit the experimental data. The relative stronger scattering of exciton with both $\mathrm{LO}$ and acoustic phonons was found in the $\beta$-GaN epilayer. Analysis shows that it is a characteristic property of $\beta$-GaN.

This project is supported by HKU Research Grant (No. 10203533) and partly supported by RGC/NSFC Joint Research Scheme (N_HKU028/00).

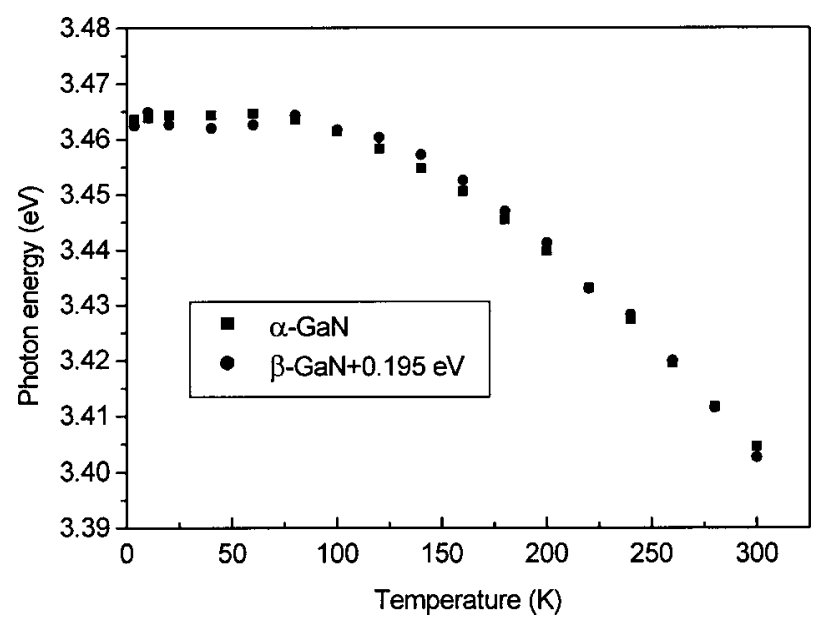

FIG. 4. Temperature dependence of the peak positions of the dominant emission for two GaN epilayers.

${ }^{1}$ S. Nakamura and G. Fasol, The Blue Laser Diodes (Springer, Berlin, 1997).

${ }^{2}$ GaN and Related Materials, edited by S. J. Pearton (Overseas Publishers Association, Amsterdam, Netherlands, 1997).

${ }^{3}$ H. Morkoç, Nitride Semiconductors and Devices (Springer, Berlin, 1999).

${ }^{4}$ See, for example, O. Brandt's recent review and references therein, in Group III Nitride Semiconductor Compounds: Physics and Applications, edited by B. Gil (Oxford University Press, London, 1998), Chap. 11, p. 417.

${ }^{5}$ J. Wu, H. Yaguchi, K. Onabe, R. Ito, and Y. Shiraki, Appl. Phys. Lett. 71, 2067 (1997).

${ }^{6}$ D. J. As, T. Simonsmeier, B. Schöttker, T. Frey, D. Schikora, W. Kriegseis, W. Burkhardt, and B. K. Meyer, Appl. Phys. Lett. 73, 1835 (1998).

${ }^{7}$ G. Mirjalili, T. J. Parker, S. F. Shayesteh, M. M. Bülbül, S. R. P. Smith, T. S. Cheng, and C. T. Foxon, Phys. Rev. B 57, 4656 (1998).

${ }^{8}$ X. L. Sun, H. Yang, L. X. Zheng, D. P. Xu, J. B. Li, Y. T. Wang, G. H. Li, and Z. G. Wang, Appl. Phys. Lett. 74, 2827 (1999).

${ }^{9}$ Z. X. Liu, A. R. Goñi, K. Syassen, H. Siegle, C. Thomsen, B. Schöttker, D. J. As, and D. Schikora, J. Appl. Phys. 86, 929 (1999).

${ }^{10}$ H. Yang, L. X. Zheng, J. B. Li, X. J. Wang, D. P. Yu, Y. T. Wang, X. W. Hu, and P. D. Han, Appl. Phys. Lett. 74, 2498 (1999).

${ }^{11}$ D. J. As, A. Richter, J. Busch, M. Lübbers, J. Mimkes, and K. Lischka, Appl. Phys. Lett. 76, 13 (2000).

${ }^{12}$ A. Rubio, J. L. Corkill, M. L. Cohen, E. L. Shirley, and S. G. Louie, Phys. Rev. B 48, 11810 (1993).

${ }^{13}$ A. F. Wright and J. S. Nelson, Phys. Rev. B 50, 2159 (1994).

${ }^{14}$ J. I. Pankove, Mater. Res. Soc. Symp. Proc. 162, 515 (1990).

${ }^{15}$ S. M. Seutter, M. H. Xie, W. K. Zhu, L. X. Zheng, H. S. Wu, and S. Y. Tong, Surf. Sci. 445, L71 (2000).

${ }^{16}$ S. Rudin, T. L. Reinecke, and B. Segall, Phys. Rev. B 42, 11218 (1990).

${ }^{17}$ I. A. Buyanova, J. P. Bergman, B. Monemar, H. Amano, and I. Akasaki, Mater. Sci. Eng., B 50, 130 (1997).

${ }^{18}$ W. Liu, M. F. Li, S. J. Xu, K. Uchida, and K. Matsumoto, Semicond. Sci. Technol. 13, 769 (1998).

${ }^{19}$ A. K. Viswanath, J. I. Lee, D. Kim, C. R. Lee, and J. Y. Leem, Phys. Rev. B 58, 16333 (1998).

${ }^{20}$ P. Perlin, C. Jauberthie-Carillon, J. P. Itie, A. San Miguel, I. Grzegory, and A. Polian, Phys. Rev. B 45, 83 (1992).

${ }^{21}$ D. C. Reynolds, D. C. Look, B. Jogai, and R. J. Molnar, Solid State Commun. 108, 49 (1998).

${ }^{22}$ S. J. Xu, C. T. Or, Q. Li, L. X. Zheng, M. H. Xie, S. Y. Tong, and H. Yang, Phys. Status Solidi A 188, 681 (2001).

${ }^{23}$ J. B. Xia, K. W. Cheah, X.-L. Wang, D.-Z. Sun, and M.-Y. Kong, Phys. Rev. B 59, 10119 (1999).

${ }^{24}$ W. Shan, B. D. Little, A. J. Fischer, J. J. Song, B. Goldenberg, W. G. Perry, M. D. Bremser, and R. F. Davis, Phys. Rev. B 54, 16369 (1996).

${ }^{25}$ B. J. Skromme, J. Jayapalan, R. P. Vaudo, and V. M. Phanse, Appl. Phys. Lett. 74, 2358 (1999).

${ }^{26}$ P. Y. Yu and M. Cardona, Fundamentals of Semiconductors: Physics and Material Properties (Springer, Berlin, 1996), p. 352.

${ }^{27}$ K. Kim, W. R. L. Lambrecht, and B. Segall, Phys. Rev. B 53, 16310 (1996). 
Applied Physics Letters is copyrighted by the American Institute of Physics (AIP). Redistribution of journal material is subject to the AIP online journal license and/or AIP copyright. For more information, see http:/ojps.aip.org/aplo/aplcr.jsp

Copyright of Applied Physics Letters is the property of American Institute of Physics and its content may not be copied or emailed to multiple sites or posted to a listserv without the copyright holder's express written permission. However, users may print, download, or email articles for individual use. 